\title{
Clinical and laboratory findings of children with identified idiopathic arthritis, investigation on treatment and prognosis - seven years experience
}

\author{
Aysun Akay Tekelı* and Ayse Oner \\ Department of Pediatric Nephrology, Dr Sami Ulus Maternity and Children's Education and Research Hospital, Turkey
}

\begin{abstract}
Objective: We aimed to compare the clinical and laboratory findings of patients with juvenile idiopathic arthritis (JIA), to determine the preferred drugs and their efficacy and to evaluate the factors affecting prognosis and development of deformity.

Methods: Retrospectively, 75 children with JIA were evaluated. Patients were divided into subgroups according to the International Association of Leagues for Rheumatology diagnostic criteria. The joints, systemic and extraarticular findings, laboratory findings, remission, deformity and relapse rates of JIA subgroups were compared.

Results: 75 patients who were diagnosed with JIA, 50.7\% were female and $49.3 \%$ were male. The largest group of patients were polyarticular JIA patients. Systemic symptoms were detected in 63 patients and extraarticular findings in 45 patients. In the systemic JIA group; anemia, leukocytosis, thrombocytosis, elevated erythrocyte sedimentation rate (ESR), C-reactive protein (CRP) and ferritin were most frequently detected. Rheumatoid factor (RF) and immunglobulin elevation were detected in the polyarticular group. Nonsteroidal anti-inflammatory drugs were started as initial treatment in 25 (33.3\%) of the patients. Combined drug treatments were applied according to the clinical status of the illness, prognostic features and treatment at the centers. The most commonly used combination was NSAID, corticosteroids and methotrexate (MTX) treatment with a rate of $29.3 \%$. The rate of remission was $85.2 \%$ while the deformity rate was $14.7 \%$ and the relapse rate was $40.4 \%$.
\end{abstract}

Conclusion: JIA is one of the most common chronic diseases of the childhood that is at the risk of sequelae and is one of the most common rheumatic diseases. In our study, the characteristics of the patients' male/female, subgroups, deformities and relapse rates were determined according to their socioeconomic and racial characteristics and clinical, laboratory features, treatments and remissions were determined.

\section{Introduction}

Juvenile idiopathic arthritis (JIA) is a chronic, systemic and inflammatory joint disease with a high risk of childhood sequelae, which is occured by various signs and symptoms, varying in the initial shape and clinical course, most common in childhood. The disease can also manifest itself as a chronic disease with chronic joint involvement as well as various organs and systemic involvement [1]. Despite the well-defined clinical manifestations of the disease, no definite diagnostic laboratory test has been found yet. If the clinical picture is not fully established, differential diagnosis with early diagnosis and other colloid tissue diseases is difficult [2]. In the absence of definitive diagnostic criteria, many classification and diagnostic criteria have been proposed according to the number of joints involved, the patient's disease, rheumatoid factor (RF) seropositivity and associated clinical findings $[1,3]$. It is now accepted that JIA is a chronic disease, as a clinically in heterogeneous arthritis, beginning before the age of 16 years according to the different symptoms that develop within the first 6 months, which are defined in a large period [4]. In this study, we aimed to determine the factors affecting the development of prognosis and deformity development according to ILAR (International Association of Leagues for Rheumatology) diagnostic criteria for children undergoing JIA diagnosis, comparison of clinical and laboratory findings, determination of preferred drugs and their efficacy, prognosis and development of deformities.

\section{Methods}

75 children with JIA according to ILAR criteria were evaluated retrospectively at Dr. Sami Ulus Children's Hospital which is a tertiary referral research unit. Patients were assessed for their gender, age that they started and symptoms began, whether they had received treatment prior to admission to the hospital, family history of kinship, number and location of joints involved, systemic findings (fatigue, loss of appetite, weight loss, growth retardation) and extraarticular findings (fever, skin rash, hepatosplenomegaly, Lymphadenopathy, rheumatoid nodule, uveitis, pericarditis), follow-up status of the patients, preferred medications in the treatment and side effects were evaluated.

According to laboratory findings Leukocytosis and thrombocytosis were defined as hemoglobin levels below 2 standard deviations according to the age of the patient, anemic white blood cells and

Correspondence to: Aysun Akay Tekel, Department of Pediatric Nephrology, Dr Sami Ulus Maternity and Children's, Education and Research Hospital, Ankara, Turkey, E-mail: aysunnakay@yahoo.com.tr

Key words: children, demographics, Juvenile idiopathic arthritis, treatment, relaps rates

Received: December 02, 2017; Accepted: December 23, 2017; Published: December 27, 2017 
thrombocytes above the upper limit determined by gender and age group. The rate of erythrocyte sedimentation (ESR) $>20 \mathrm{~mm} / \mathrm{h}$, $\mathrm{C}$ reactive protein $(\mathrm{CRP})$ is considered to be higher than $5 \mathrm{mg} / \mathrm{L}$. Ferritin was studied with nephelometric methods and was accepted as normal between 7-140 $\mathrm{ng} / \mathrm{ml}$ [5]. Rheumatoid factor (RF) was determined by latex agglutination method and nephelometric method. Bone mineral dasitometry was assessed using long bone and all joint graphs and DEXA method. Immunoglobulin levels were determined by immunonephelometric method and found to be above the age and sex defined limits [5].

Our patients were evaluated according to the presence or absence of poor prognostic criteria. Persistent RF positivity, radiological narrowing or erosion of joint space, polyarticular synovitis, nonsteroidal treatment for one year and dramatic improvement in combination with methotrexate, intraarticular steroid or slow acting antirheumatic drugs, HLA-DR4 positivity in patients with polyarticular arthritis, the prognosis was considered to be a poor prognostic criterion because of the presence of polyarthritic JIA, systemic JIA with polyarticular involvement, deep anemia accompaniment, presence of hypergamaglobulinemia, and at least 2 cases of very high ESR [6]. Remission is accepted as our patient hadn't morning stiffness that did not last longer than 15 minutes, fatigue, absence of soft tissue swelling and joint sensitivity, absence of motion, pain and limitation of motion, and ESR of less than $30 \mathrm{~mm} / \mathrm{h}$ were considered as 5 or more.

In addition to clinical findings in deformed joints, joint space narrowing, articular surface erosion, subcortical cysts, ankylosis and fusion formation were identified by radiographic findings.

\section{Statistical analysis}

In the comparison of the ratios of the obtained data, "significance test of the difference between the percentages in the independent groups" was used. The "Fischer exact and chi-square test" was used to determine the significance of the difference between observed frequencies and expected frequencies. "Kruskal Wallis test" was used in the intergroup comparisons. SPSS (Statistical Package for Social Sciences) for Windows 15 was used for statistical analysis and $\mathrm{p}<0.05$ was accepted meaningful.

\section{Results}

In the 7-year period of this tertiary education and research hospital in the Department of Nephrology who were diagnosed with JIA, 38 (50.7\%) were girls and of 37 (49.3\%) were boys and the girl/boy ratio was found as 1.03. Age average of the majority of patients according to onset of symptoms was between 2 and 7 years (42.7\%). When the cases were classified according to clinical findings in the first six months, the largest group consisted of 49 patients (65.3\%) with polyarticular JIA, $48 \%$ of them were RF (-) and $17.3 \%$ were RF (+) patients. Thirteen patients (17.3\%) with systemic onset JIA, 12 (16\%) with oligoarticular JIA and 1 patient $(1.3 \%)$ with associated JIA were detected. Age of onset of the disease were found as $7.6 \pm 3.40$ in the polyarticular RF group, $10 \pm 2.86$ in the RF (+) group, $8.5 \pm 3.48$ in the oliartic group and $4 \pm 4.15$ in the systemic group.

Mean time between onset of symptoms and admission to hospital were 16.5 months in polyarticular RF (-) group, 4 months in RF (+) group and 6 months in oligoarticular and systemic group. In 21 of our patients (28\%) kinship was detected, $57.1 \%$ of them were first-degree relatives.

Arthritis was the most common finding and arthritis was present in all groups in the course of application. In all patients, limitation of motion was detected in 58 patients $(77.3 \%)$, temperature increase in 29 patients (38.7\%), redness in 23 patients (30.7\%) and stiffness in 35 patients $(46.7 \%)$. The most frequently involved joint was found to be the knee joint with $88 \%$ frequency, the ankle, fingers, wrists and toes with decreasing frequency.

Extraarticular findings were detected in 45 (60\%) patients. Fever was the most common finding in all patients in the systemic group, although 18 (36.7\%) patients were found in the polyarticular group and no cases were found in the oligoarticular group patients.In the systemic JIA group,rheumatoid skin rash was detected in $6(42.6 \%)$ patients and in lymphadenopathy in $4(30.8 \%)$ patients. The rate of rheumatoid nodule in the polyarticular group was $4.1 \%$. Uveitis was the most common in $3(25 \%)$ patients in the oligoarticular group. Pericarditis 3 (4\%) was detected in the patient. Hepatomegaly, splenomegaly and hepatosplenomegaly were detected in the most systemic group, 7 (53.8\%), $4(30.8 \%)$ and $4(30.8 \%)$ patients, respectively.

When laboratory findings of the cases are evaluated anemia was found in $76.9 \%$, leukocytosis in $38.5 \%$ and thrombocytosis in $76.9 \%$. ESR and CRP were very high in all patients with systemic JIA. Ferritin was detected again in $70 \%$ of patients with the most frequent systemic JIA and $50 \%$ with polyarticular JIA. Immunoglobulin elevation was detected in the polyarticular and systemic JIA groups.

68 patients who underwent direct radiography were evaluated by a radiologist. 12 normal finding, 27 soft tissue swelling, 26 osteopenia and 15 osteoporosis were detected in patients. Bone mineral densitometry (BMD) was performed on 31 of our patients and osteoporosis was detected in $18 / 47(38.3 \%)$ patients in the polyarticular group, $6 / 12$ (50\%) in the oligoarticulargroup and $7 / 11(63.6 \%)$ in the systemic group. Of 31 patients with osteoporosis, 28 (90.3\%) received steroid treatment.

Disease treatment was began as taking into consideration the subgroup characteristics of the disease, the severity of the joint and systemic findings of the patient, the duration of the illness, the medications used, the response provided by these medications and the presence of poor prognostic criteria. 25 patients (33.3\%) took nonsteroidal anti-inflammatory drug (NSAID) alone, Naproxen and Tolmetin were the most commonly used. Sulfosalazine, methotrexate (Mtx) and steroid treatments were added to the treatment of 12 patients who could not receive NSAID treatment. Steroids, methotrexate and other slow-acting antirheumatic drugs were added to the patient's NSAIDs at the time of admission, limited mobility, multiple poor prognostic criteria, or treatment at the external centers. Cytotoxic agents were administered to 5 patients. 4 patients were systemic JIA, 1 patient was oligoarticularJIA group with uveitis.

Side effects were observed in 33 (44\%) patients due to the drugs used. Cataract, glaucoma in 1 patient and cataract and glaucoma in 1 patient developed in 5 patients who tooksteroid treatment, osteoporosis was detected in 23 patients, and bisphosphonate, calcitonin, vitamin D and/ or calcium treatment were given to these patients.

Cushingoid appearance in steroid-induced 16 patients, increase the virilisation in 3 patients, acne in 2 patients, myopathy in 1 patient, muscle atrophy in 1 patient, triglyceride and cholesterol uptake in 1 patient, blood sugar and elevation in 1 patient and depression in 1 patient were detected. In these patients, diet and medication dose adjustments were made. Anemia in 4 patients receiving methotrexate, leucopenia in 2 patients, pancytopenia in 1 patient, and pancytopenia due to cyclosporin-A in 1 patient were discontinued. Elevation of the liver function tests of 2 patients treated with Mtx and 1 patient using 
naproxen was detected and the treatment was interrupted for a while. Drug reduction has been reduced on the development of nausea in a patient receiving Mtx treatment. In patients with oligoarticular JIA who had begun cyclosporine-A due to uveitis, there was slight deterioration in kidney function tests and the patient was offered abundant hydration. In a patient who received Tolmetin, proteinuria developed, but it was determined that there was a pseudopregnant proteinuria.

64 (85.3\%) of the patients regularly used their medication. $85.7 \%$ of patients with polyarticular JIA, $83.3 \%$ of patients with oligoarticular JIA, and $84.6 \%$ of patients with systemic onset JIA had regularly used their medication. Of the 75 patients, $61(81.3 \%)$ had follow-up. The follow-up duration of the patients was at least 6 months, maximum 7 years, and average 1.5 years.

$1(1.6 \%)$ patient with systemic onset JIA amyloidosis died subsequently developed pancytopenia and sepsis after 2 years of follow-up.

Deformity developed in $9(14.7 \%)$ of the patients and 8 (20.5\%) in the polyarticular group and $1(10 \%)$ in the systemic onset group. There was no development of deformity in the oligoarticular group and intracerebral JIA patient. The developing deformities were knee,wrist, flexion contracture of the fingers and ulnar deviation.

Twenty-one patients (40.4\%) developed relapses among the 52 patients who were followed-up. There were 12 (37.5\%) relapses in the polyarticular group, $2(18.2 \%)$ in the oligoarticular group and 7 (87.5\%) patients in the systemic onset group.

\section{Discussion}

Juvenile idiopathic arthritis (JIA) is a childhood disorder characterized by chronic synovitis and various subgroups. Despite being a long-known disease, etiopathogenesis has not been explained completely. Various publications concerning JIA indicated that the initial findings, clinical course, and laboratory findings of the disease are very variable $[7,8]$. The epidemiological studies on JIA are based on the distribution of the disease and the subgroup characteristics varying in different ethnic groups and socioeconomic levels.

In this study, we evaluated the epidemiological, clinical, laboratory findings, treatment and prognostic features of patients who were diagnosed with JIA in our hospital. When all types of the disease are considered, the ratio of Girls / Boys is $2 / 1$, and oligoarticular type is 3 times more than girls. There is no gender difference in the systemic onset type (G / B: 1/1) [6]. In a study that performed about JIA in our country, male patient dominance was found [9]. Of the 75 patients included in our study, 38 (50.7\%) were girl and 37 (49.3\%) were boy and the ratio of girl to boy was 1.03. Contrary to classical information, the ratio of Girls / Boys was equal to each other. It can be inferred that boys may have been brought to reference centers more frequently than girls since boys are much more in our society. According to previous studies, the ratio of girls / boys in our study is equal to that of girls and it can be explained by the increase in socio-cultural level and bringing them to advanced health institutions. In our study, there were more girls in the systematic and polyarticular JIA group while the boys in the oligoarticular JIA group were found much more.

Although the disease may occur at any age in childhood, age distribution according to sex and subgroups is reported differently by various researchers. The symptoms are observed most frequently under 5 years, especially between 1 and 3 years and increase slightly again towards puberty. This distribution is more pronounced in girls and in oligoarticular cases [3]. In males, the peak is around 2 and 9 years [10]. In systemic onset JIA, the age onset of the disease is less obvious [6]. In our study, it was determined that $42.7 \%$ of the patients were between 2 and 7 years of age. These results are consistent with other studies $(17,23)$. In our study, the second age group that the male patients most frequently concentrated on was found to be the 8-11 age groups. In girls, the age of onset of the disease was found to be approximately equal between 2-7 and 8-11 years of age. These results were consistent with the literature $[6,10]$.

Of the 75 patients included in our study, $65.3 \%$ had polyarticular JIA patients whereas $16 \%$ had oligoarticular JIA patients, $17.3 \%$ had systemic onset JIA patients, and $1.3 \%$ was associated with intensity JIA patients.

The RF (-) polyarticular group accounts for $48 \%$ and the RF (+) group accounts for $17.3 \%$. Kasapçopar Ö. and his colleagues did a study on the subgroups of JIA, (35.6\%), RF (-) polyarticular group, $15.6 \% \mathrm{RF}(+)$ group, $17.3 \%$ persistentoligoarticular group, $15.6 \%$ prolonged oligoarticular group and $15.6 \%$ systemic onset group [11]. The ratios in this study are consistent with the results of our study. However, the high proportion of polyarticular JIA patients in our study may be attributed to the fact that the patient's age between admission to the hospital and the onset of symptoms was long and may have been included in the polyarticular subgroup of patients with prolonged oligoarticular JIA.In the literature, it has been reported that $30-60 \%$ of JIA produces oligoarticular, $20-40 \%$ polyarticular, $5-20 \%$ systemic onset type and $1-11 \%$ incidence-related arthritis [4). In their series, Schaller and Wedgwood detected $37 \%$ of polyarticular and oligoarticular types and $26 \%$ of systemic type [12]. In the studies it is found that polyarthritis subtype is common in Costa Rica, India, New Zealand, and South Africa, although oligoarticular subtype is more prevalent in Western European countries [13,14]. In North America and Europe, oligoarticular JIA was the most common, followed by polyarticular JIA and least frequently seen is systemic JIA $[15,16]$. The appearance in Asia is quite different. The oligoarticular group is less common. In Fujikawa and Okuni's study of 570 JIA children in Japan, $54 \%$ of patients with systemic onset JIA, $25 \%$ with polyarticular JIA and $21 \%$ with oligoarticular JIA were found [17]. In an Indian study, $14 \%$ and $24 \%$ of patients with systemic onset JIA, $52 \%$ and $46 \%$ polyarticular JIA, 35\% and 30\% oligoarticular JIAwere detected [18]. Compared with the results in these studies, the difference in the rates of subgroups of the disease in our study may be due to our racial and genetic characteristics. The butcher and his colleagues support our work in this direction on the findings of his work.

The age groups frequently seen in subgroups of the disease vary. Early and late onset types of polyarticular JIA have been reported while oligoarticular JIA begins at an early age. It has been reported that an early-onset type occurs before the age of 8 , and a late-type type occurs after the age of 8 . Late-onset polyarticular JIA is usually seen with $\mathrm{RF}(+)$. In systemic onset JIA, the age at onset of the disease is less obvious [16]. In our study, the average age of onset of illness; $7.9 \pm$ 3.40 in the polyarticular RF (-) group, $10 \pm 2.86$ in the polyarticular $\mathrm{RF}(+)$ group, $8.5 \pm 3.48$ in the oligoarticular group and $4 \pm 4.15$ in the systemic group. In a study of 67 JIA ILAR classifications in France, in the systemic onset group age of onset the symptoms weredetected as 4.2 in the oligoarticular group and 6.7 in the RF (-) polyarticular group [19]. They reported that the systemic type started at an early age, whereas oligoarticular and polyarticular types appeared at a later age. These results are consistent with our study. 
In JIA, the most common finding of the disease is joint pain and swelling in the joints. Restraint of mobility may be accompanied by pain or tenderness, increased temperature locally and redness. Arthritis was initially present in all of our patients. Polyarticular JIA is characterized by involvement of five or more joints. This type of arthritis tends to be symmetrical and can involve any joint in the body. In the hands, small joints, especially proximal interphalangeal joints, metacarpophalangeal joints and wrists are characteristic. It is similarly affected by small joints. Large joint involvement; knee, elbow, ankle joints are often. In oligoarticularJIA, large joints are involved, and knee, elbow, hand and ankle joints are the most frequently affected as synovial structures. Joint involvement in systemic JIA is similar to other subgroups $[4,10,20]$. In our study, knee and ankle joint involvement was most frequently seen in parallel with this information. In all JIA groups, knee involvement is seen as $88 \%$ followed by ankle $80 \%$, hand fingers $61.3 \%$ and wrist $52 \%$ frequently.

The frequency of joint involvement in the polyarticular group $91.8 \%$ of the knees; the most common, $85.7 \%$ of the ankles, $73.4 \%$ of the fingers and $55.1 \%$ of the wrists were found.It is observed that in the oligoarticular group, $83.3 \%$ knee joint and $41.7 \%$ ankle involvement. These two joints were the most commonly involved joints in the oligoarticular group. In all patients with systemic onset JIA, ankle involvement was detected in $92.3 \%$ of the cases, wrists and toes in $76.9 \%$, and knee and fingers in $76.9 \%$ of cases. There is no proportion about involvement joints in the literature; these findings are consistent with classical information about joint regions in JIA $[1,3,10,13]$.

In the literature, it has been reported that hand and toe finger involvement is more frequent in polyarticularand there isn't given proportion. Systemic onset JIA large and small joints usually involve as polyarticular. In our patients, hand fingers were affected by $61.3 \%$ and toes were affected by $48 \%$. Poliarticula JIA showed that hand fingers were affected by $73.5 \%$ and toes were affected by $48.9 \%$. According to a study done, this rate was found to be higher [10].

The involvement of the hands and toes was higher in patients with systemic onset JIA. In our study, $92.3 \%$ of toes and $76.9 \%$ of fingers were held in this subgroup. Systemic onset JIA may have transient arthritis findings during periods of increased fever in almost all children. With fever, these findings disappear and the patient's clinic is dramatically improved. The presence of hand and toe involvement in such cases may be due to the evaluation of arthritis as a temporary joint finding in the acute phase of systemic disease that lasts for 6 weeks.

In JIA, fever can be observed in about one third of patients. One of the most common findings in the systemic groupis intermittent fever that rise up to $39.5^{\circ} \mathrm{C}, 1-2$ times a day.

Fever may also be detected in the polyarticular group, but is less common in the oligoarticular group. Fever in the polyarticular group was reported as $30 \%$ and in the systemic group as $100 \%$ [13]. In our study, fever was observed in all of the patients with systemic onset JIA, while in the polyarticular group it was $36.7 \%$. Patients in the oligoarticular group had no fever. These findings were consistent with the literature.

Rheumatoid rash is one of the classic findings of systemic JIA. Patients may have a solitary pink, pale, usually white, macular debris that can appear anywhere on the body, with fever elevation. It is less common in polyarticular and oligoarticular groups. A rheumatoid rash was detected in $16 \%$ of our cases. The rate of systemic onset JIA was $42.6 \%$, polyarticular JIA was $10.2 \%$, oligoarticular JIA was $8.3 \%$. In literature this rate is $95 \%, 2 \%, 0 \%$ respectively [21]. In a study conducted, rheumatoid rash was detected in $18.2 \%$ of all JIA subgroups [9]. The low rate of rheumatoid rash in systemic onset JIA can be attributed to the fact that our patients and families do not notice or worry about spillage.

In literature, the incidence of rheumatoid nodules is between $5-10 \%[3,13]$. Nodules are usually reported in the polyarticular group. In our study, rheumatoid nodule was not seen in the systemic onset and oligoarticular group, but $4.1 \%$ in the polyarticular group. These ratios are compatible with the literature.

Chronic uveitis especially affects girls with oligoarticular JIA. This rate is $20 \%$ in the oligoarticular group, $5 \%$ in the polyarticular group and $1 \%$ in the systemic onset group $[3,4,13]$. Chronic uveitis rate was detected in 3 patients (25\%) with oligoarticular JIA and 2 patients (4.1\%) with polyarticular JIA. These rates were consistent with the literature [22]. Chronic uveitis was detected in 1 patient with systemic onset JIA.

Pericarditis is frequent in systemic onset JIA. It is usually asymptomatic, and some children may have dyspnea or precordial pain. The literature reports $35-73 \%$ in the systemic onset group, $5 \%$ in the polyarticular group and $0 \%$ in the oligoarticular group and 3-9\% in all JIA patients $[3,13,23]$. Lietman and colleagues found a pericarditis ratio of 7\%, but in postmortem studies this ratio increased to $45 \%$ (24). In our cases, pericarditis was detected in 1 patient with systemic onset JIA (7.7\%), 2 patients with polyarticular JIA (4\%), but oligoarticular was not detected in JIA patients. All of our JIA patients were detected at $4 \%$. These rates in our study were found to be consistent with the literature.

Generalized lymphadenopathy (LAP), polyarticular is reported as $5 \%$ in patients with JIA and $70 \%$ in patients with systemic onset JIA. The most commonly involved parts are the anterior cervical, axillary and inguinal lymph nodes [13]. 4 of the patients in the generalized LAP systemic group (30.8\%) and 3 of the patients in the polyarticular group (6.1\%) were detected. $9.3 \%$ of all JIA findings had generalized LAP.

Hepatosplenomegaly most commonly occurs in the systemic onset group, which may occur within a few years after the onset of the disease [5]. In the literature, $85 \%$ of systemic onset JIA, $10 \%$ of polyarticular JIA, and $0 \%$ of oligoarticular JIA are reported [13]. In our cases hepatosplenomegaly was detected in $4(30.8 \%)$ patients in the systemic onset group and $3(6.1 \%)$ in the polyarticular group, but not in the oligoarticular group. The low ratio of generalized LAP and hepatosplenomegaly in systemic onset patients may be depend on the earlier treatment of these patients.

Anemia in JIA is a common finding especially in systemic patients, but is common in all forms of JIA. It is usually due to chronic disease and serum iron, iron binding capacity decreases. Serum ferritin levels may increase due to disease activity. Gastrointestinal blood loss due to NSAID intake or iron deficiency anemia may also be observed due to underlying absorption and underlying inflammatory disease and malfunction in use. $41.4 \%$ of our oligoarticular JIA patients had mild iron deficiency anemia, $71.4 \%$ of mild-to-moderate anemia in our polyarticular JIA patients, and $76.9 \%$ of moderate to severe anemia in our systemic JIA patients. It is thought that serum ferritinlevels were found to be $53.6 \%$ higher in our observable patients. Ferritin levels are seen due to disease activity. These findings are consistent with classical information $[3,10]$.

Leukocytosis is seen frequently in systemic onset JIA. Leukocytosis rate in systemic JIA is reported as $80 \%$ in the literature. Leukocytosis 
was detected in 10 of our 75 patients (13.3\%). Systemic JIA was the most common with $38.5 \%$. The reason for this low rate can be attributed to the fact that $53.3 \%$ of our patients had been treated at the centers before applying to our hospital.

Thrombocytosis is usually seen in patients with systemic onset and in the polyarticular group [13]. Thrombocytosis was detected in $72 \%$ of our patients. It was found to be $76.9 \%$ in patients with systemic onset JIA, $73.4 \%$ in patients with polyarticular JIA, and $58.3 \%$ in patients with oligoarticular JIA, consistent with classical information [5].

Erythrocyte sedimentation rate (ESR) is useful following active disease and treatment. CRP is used in the same way. However, CRP is less affected than anemia and other factors [5]. ESR elevation in $94.7 \%$ of our patients and CRP increase in $93.2 \%$ of our patients were detected.

These findings are consistent with the literature [13].

In JIA patients, RF (+) is reported in 5-25\% (9). In our cases, RF was examined with latex fixation technique. RF positivity was detected as $17.3 \%$. In the polyarticular group $26.5 \%$ were detected, but no RF positivity was detected in the oligoarticular and systemic onset groups. Our results were consistent with the literature $[1,3,13]$.

In studies conducted, Ig levels were found to be high in 6-37\% of patients $[25,26]$. It has been reported that Ig increase is observed especially in the systemic and polyarticular group.

JIA is a systemic disease that causes osteoporosis [27]. Radiological examinations are used to evaluate the pathologies related to the disease, to evaluate the complications related to the disease and treatments used. In Brazil, a 50-60\% reduction in bone density in the lumbar vertebra and femur neck was found [3]. Osteoporosis was detected in $18(38.3 \%)$ patients with polyarticular JIA, $6(50 \%)$ patients with oligoarticular group and 7 (63.6\%) patients with systemic onset group who were not studying bone grafts and bone mineral densitometry studies. Totally in 31 patients $(43.7 \%)$ osteoporosis was detected. These ratios are compatible with the literature. $90.3 \%$ of our patients diagnosed with osteoporosis were receiving steroid treatment. It is detected that steroids increase the frequency of osteoporosis according to our hospital and abroad studies $[28,29]$.

Nonsteroidal anti-inflammatory drugs (NSAIDs) are the basis of treatment in a large majority of patients in JIA. It is used in initial treatment in many children. NSAID therapy was initiated onlyin 25 of our patients (33.3\%). Slow effective antirheumatic and immunosuppressive drugs were added to NSAIDs in $50(66.7 \%)$ patients with poor prognostic criteria and various treatments at the external center. The most preferred NSAIDs are Naproxen and Tolmetin. Tolmetin in 5 patients $(20 \%)$ and Naproxen therapy were given only in 20 patients (80\%). Tolmetin treatment only was given to Naproxen in 11 (55\%) of 20 patients who started Naproxen and Tolmetin treatment in 2 (40\%) of 5 patients who started Tolmetin. However, 7 of these patients did not come to the controls.

There was no need to add slow-acting antirheumatic and immunosuppressive therapy to the treatment of 6 patients who only received NSAIDs in the follow-up patients. NSAIDs were in the oligoarticular and polyarticular groups of our patients. Only 5 of our NSAID-treated patients received remission. In 1 patient, treatment change was not considered because he did not use the drug regularly. In the literature Tolmetin activity is reported in $50-80 \%$. Tolmetine was found in $25 \%$ and $86 \%$ of the other two studies $[30,31]$. The response to Naproxen varies from $24 \%$ to $89 \%$ [32]. In our study, the efficacy ratios of NSAID drugs were found to beconsistent with the literature.
Early intervention of slow acting antirheumatic and immunosuppressive drugs in addition to NSAIDs is recommended in patients with prolonged active joint involvement or poor prognostic criteria. In this way, the active inflammatory process is stopped as soon as possible and the patients enter the remission rapidly [33].

Although steroids have limited use in JIA, they are recommended for use in life-threatening conditions or severe joint involvement [33]. It was learned that 39 (52\%) patients with poor prognostic factors and steroid treatment in the external centers were given steroids in the initial treatment. According to clinical and laboratory findings, we have added steroid treatment for our following 10 patients. (pulse methylprednisolone or oral prednisolone) as well as treatments that they took.

If it is failed response to NSAID therapy it is recommended for treatment of methotrexate as a second option $[34,35]$.

In the study performed by Giannini and his colleagues, the effectiveness of the method was investigated. In this study, one of the groups was added to the treatment of patients with $5 \mathrm{mg} / \mathrm{m}^{2} /$ week methotrexate and $10 \mathrm{mg} / \mathrm{m}^{2} /$ week as the second drug. These two groups were compared with the placebo group without taking 2nd drug treatment.Methotrexate was given to 38 of our follow-up patients (62.3\%). Mtx was used at a dosage of 7.5-10 $\mathrm{mg} / \mathrm{m}^{2} /$ week. This dose is consistent with classical information [36].

Remission was seen in $33(86.8 \%)$ of our Mtx-treated patients. Remission was observed in 6 (85.7\%) of 7 systemic onset patients using Mtx $22(84.6 \%)$ of 26 patients in the polyarticular group and 5 in the oligoarticular group (100\%).

The remission rate was found low because patients in the polyarticular group receiving Mtx therapy did not use their medications regularly and the remission rate with Mtx treatment was found to be quite high in systemic onset patients. Mtx therapy should be recommended in patients with poor prognostic factors, such as polyarthritis and systemic onset who are not responding to NSAIDs in the presence of these findings.

Sulfosalazin was given to 16 (26.2\%) of our follow-up cases. 8 of these patients were treated with NSAID, 8 with NSAID and steroid treatment with sulfosalazine treatment. Remission was detected in 6 (75\%) patients receiving NSAID + sulfosalazine treatment. Remission was observed in each 1 patient with oligoarticular systemic onset amongst 4 of 6 patients 66.7\%) in polyarticular group.In a study conducted by Van Rossum and his friends, one group of patients in the oligoarticular and polyarticular group was treated with sulfosalazine and the other group with placebo. Sulfosalazine $50 \mathrm{mg} / \mathrm{kg} /$ day was used. The activity was detected as $44 \%$ which was found meaningful compared to the placebo group [37].

Seven of the 8 patients $(87.5 \%)$ who received NSAID + sulfosalazine + steroid treatment had remission. No remission was observed in 1 patient in the systemic baseline group with poor prognostic factors and cyclosporine treatment was added to the patient's treatment.

Slow-acting antirheumatic medications such as Hydroxychloroquine, D-penicillamine, gold salts and other new treatment approaches have not been used in our patients.

The prognosis of the disease varies depending on the clinical course of the disease, its complications, and the selected treatment response. Juvenile idiopathic arthritis is the major cause of morbidity, chronic arthritis in the polyarticular and systemic onset group, and iridocyclitis 
in the oligoarticular group [3]. The prognosis is different in each patient. Despite the fact that it is a chronic illness and not a definite specific treatment, it is benign and usually there is no life threatening even in severe systemic involvement. Although the prognosis for RF (+) polyarticular type and systemic onset type is poor in terms of joint function, the general prognosis of the disease is good and $75-80 \%$ of patients enter remission [1]. When we evaluated the remission rates of our patients, $82 \%$ of the patients in the polyarticular group and $80 \%$ of the systemic-onset patients were admitted to remission while all patients in the oligoarticular group entered the remission. In our follow-up cases, the remission rate was $85.2 \%$. This ratio was found to be consistent with the literature [10].

Systemic onset and RF (+) cases in JIA are at risk with regard to joint destruction. Severe arthritis in the systemic onset JIA is $30-50 \%$, in the $\mathrm{RF}(-)$ polyarticular group is $10-15 \%$, in the $\mathrm{RF}(+)$ polyarticular group is $50 \%$, and when severe functional limitation is reported as $25 \%$ in all JIA patients $[4,10,38]$. In our present cases, the deformity rate was found as $14.7 \%$. 20.5\% in the polyarticular group and $10 \%$ in the systemic onset group, whereas no deformity developed in any of the patients in the oligoarticular group. A low detection of our rate of deformity can be attributed to our racial and genetic characteristics.

The frequency of recurrence and active arthritis recurrence in JIA is quite high. If there is poor prognosis criteria for treatment and if there is rapid progression of arthritis, it is suggested to reverse the treatment pyramid, start the Mtxand other second-line drugs immediately.

Relapses can occur after remitting in JIA patients. Relapse has been reported in $50 \%$ of patients with systemic onset JIA [38]. The relapse rate was $40.4 \%$ in our patients. The most frequent relapse was seen in $87.5 \%$ of systemic onset JIA, in $37.5 \%$ of polyarticular group and in $18.2 \%$ of oligoarticular group. Relapse rates may be due to racial and genetic differences and treatment compliance.

Macrophage activation syndrome (MAS), which is rarely seen in $5-8 \%$ of systemic onset JIA, is a life-threatening complication [39]. MAS developed in 1 of our systemic onset JIA patients (7.8\%). This ratio was found to be consistent with the literature.

Juvenile idiopathic arthritis is rarely fatal. Mortality rate was reported as $0,1 \%$ [20]. In systemic onset disease, $4 \%$ of children die from amyloidosis and infection. The most common cause of death in patients with renal disease was amyloidosis $[38,40]$.

In our cases, one patient died from pancytopenia and sepsis from the systemic onset JIA group. Our patient was on steroid treatment and amyloidosis was detected. The mortality rate in all patients was found as $1.3 \%$.

Amyloidosis was detected in 3 of our patients (4\%). The rate of amyloidosis in the literature is reported between 1-10\% [38,41]. This rate was found to be compatible with our study.

In juvenile idiopathic arthritis which is a chronic disease, compliance with follow-up and treatment of patients is very important. JIA is a disease that is difficult to diagnose and treat, and should be followed for life. Despite high rates of recurrence, patients with delayed diagnosis and started to treatment lateoccur at high rates of deformity and their standard of living declines. Apart from drug treatment, patients should be provided with physical and occupational therapy and psychosocial support treatments. JIA treatment is a team work that includes children, family, pediatric rheumatologist, physical therapist, physiotherapist, psychologist, ophthalmologist, dentist, orthopedist, nurse, occupational therapist, nutritionist and social workers. All team members must work in conjunction with each other.

\section{Conclusion}

As we have shown in our study, the treatment of nonsteroidal antiinflammatory drugs and steroids used in the early period of the disease was an effective treatment. Especially the addition of methotrexate and disease-slowing antirheumatic drugs could stop the progress of the disease and can prevent permanent joint damage and uveitis.

\section{References}

1. Woo P, Wedderburn LR (1998) Juvenile chronic arthritis. Lancet 351: 969-973. [Crossref]

2. Huppertz HI (1993) Viewpoint: time for change in pediatric rheumatology. Rheumatology international 13: 37-38.

3. Schaller JG (1997) Juvenile rheumatoid arthritis. Pediatrics in review. American Academy of Pediatrics 18: 337.

4. Ravelli A1, Martini A (2007) Juvenile idiopathic arthritis. Lancet 369: 767-778 [Crossref]

5. Kliegman RM, Stanton B, Geme JS, Schor NF, Behrman RE (2015) Nelson textbook of paediatrics. Elsevier Health Sciences.

6. Cassidy J, Petty RE (2001) Juvenile rheumatoid arthritis. Textbook of pediatric rheumatology 4: 218-322.

7. Tzoufi M, Siamopoulou-Mavridou A, Challa A, Lapatsanis P (1994) Changes of mineral metabolism in juvenile chronic arthritis. Acta Paediatr Suppl 83: 52-57. [Crossref]

8. Fink CW, Fernandez-Vina M, Stastny P (1995) Clinical and genetic evidence that juvenile arthritis is not a single disease. Pediatr Clin North Am 42: 1155-1169. [Crossref]

9. Bas AY (2001) Jüvenil Romatoid Artritli Hastalarin Incelenmesi.Dr. Sami Ulus Çocuk Sagligi ve Hastaliklari Merkezi. Ankara Uzmanlik tezi.

10. Weiss JE, Ilowite NT. Juvenile idiopathic arthritis. Rheumatic Disease Clinics of North America. 2007;33(3):441-70. [Crossref]

11. Kasapçopur Ö, Demirli N, Özdogan H, Apelyan M, Çaliskan S, et al. (2005) Evaluation of classification criteria for juvenile-onset spondyloarthropathies. Rheumatol Int 25: 414-418. [Crossref]

12. Schaller J, Wedgwood RJ (1972) Juvenile rheumatoid arthritis: a review. Pediatrics 50 940-953. [Crossref]

13. Cassidy JT, Petty RE, Laxer RM, Lindsley CB (2010) Textbook of pediatric rheumatology: Elsevier Health Sciences.

14. Szer IS, Kimura Y, Malleson PN, Southwood TR (2006) Arthritis in children and adolescents: juvenile idiopathic arthritis: Oxford University press USA.

15. Bowyer S, Roettcher P (1996) Pediatric rheumatology clinic populations in the United States: results of a 3 year survey. Pediatric Rheumatology Database Research Group. $J$ Rheumatol 23: 1968-1974. [Crossref]

16. Symmons DP, Jones M, Osborne J, Sills J, Southwood TR, et al. (1996) Pediatric rheumatology in the United Kingdom: data from the British Pediatric Rheumatology Group National Diagnostic Register. J Rheumatol 23: 1975-1980. [Crossref]

17. Fujikawa S, Okuni M (1997) Clinical analysis of 570 cases with juvenile rheumatoid arthritis: results of a nationwide retrospective survey in Japan. Acta Pediatr Jpn 39: 245-249. [Crossref]

18. Seth V, Kabra S, Semwal O, Jain Y (1996) Clinico-immunological profile in juvenile rheumatoid arthritis — an Indian experience. Indian J Pediatr 63: 293-300. [Crossref]

19. Danner S, Sordet C, Terzic J, Donato L, Velten M, et al. (2006) Epidemiology of juvenile idiopathic arthritis in Alsace, France. J Rheumatol 33: 1377-1381. [Crossref]

20. Gallagher KT, Bernstein B (1999) Juvenile rheumatoid arthritis. Curr Opin Rheumatol 11: 372-376. [Crossref]

21. Petty RE, Southwood TR (1998) Classification of childhood arthritis: divide and conquer. J Rheumatol 25: 1869-1870. [Crossref]

22. Bowyer SL, Roettcher PA, Higgins GC, Adams B, Myers LK, et al. (2003) Health status of patients with juvenile rheumatoid arthritis at 1 and 5 years after diagnosis. $J$ Rheumatol 30: 394-400. [Crossref] 
Tekelı AA (2018) Clınıcal and laboratory findıngs of chıldren with ıdentıfied ıdıpathıc arthritıs, investıgation on treatment and prognosıs - seven years experıence

23. Glass DN, Giannini EH (1999) Juvenile rheumatoid arthritis as a complex genetic trait. Arthritis \& Rheumatism 42: 2261-2268.

24. Lietman PS, Bywaters EG (1963) Pericarditis in Juvenile Rheumatoid Arthritis. Pediatrics 32: 855-860. [Crossref]

25. Gutowska-Grzegorczyk G, Baum J (1976) Serum immunoglobulin and complement interrelationships in juvenile rheumatoid arthritis. $J$ Rheumatol 4: 179-185. [Crossref]

26. Cassidy JT, Petty RE, Sullivan DB (1973) Abnormalities in the distribution of serum immunoglobulin concentrations in juvenile rheumatoid arthritis. J Clin Invest 52: 1931. [Crossref]

27. Moore TL (1999) Immunopathogenesis of juvenile rheumatoid arthritis. Curr Opin Rheumatol 11: 377-383. [Crossref]

28. Cassidy JT, Hillman LS (1997) Abnormalities in skeletal growth in children with juvenile rheumatoid arthritis. Rheum Dis Clin North Am 23: 499-522. [Crossref]

29. Athreya BH (1996) Management of rheumatic diseases in children. Indian J Pediatr 63 305-321. [Crossref]

30. Levinson JE, Baum J, Brewer E, Fink C, Hanson V, et al. (1977) Comparison of tolmetin sodium and aspirin in the treatment of juvenile rheumatoid arthritis. J Paediatr 91: 799-804. [Crossref]

31. Leak A, Richter M, Clemens L, Hall M, Ansell B (1987) A crossover study of naproxen, diclofenac and tolmetin in seronegative juvenile chronic arthritis. Clin Exp Rheumatol 6: 157-160. [Crossref]

32. Ruperto N, Nikishina I, Pachanov ED, Shachbazian Y, Prieur AM, et al. (2005) A randomized, double-blind clinical trial of two doses of meloxicam compared with naproxen in children with juvenile idiopathic arthritis: Short-and long-term efficacy and safety results. Arthritis Rheum 52: 563-572. [Crossref]
33. Giannini EH, Cawkwell GD (1995) Drug treatment in children with juvenile rheumatoid arthritis. Past, present, and future. Pediatr Clin North Am 42: 1099-1125. [Crossref]

34. Hashkes PJ, Laxer RM (2005) Medical treatment of juvenile idiopathic arthritis. JAMA 294: 1671-1684. [Crossref]

35. Cassidy JT (1999) Medical management of children with juvenile rheumatoid arthritis. Drugs 58: 831-850. [Crossref]

36. Giannini EH, Brewer EJ, Kuzmina N, Shaikov A, Maximov A, et al. (1992) Methotrexate in resistant juvenile rheumatoid arthritis: results of the USA-USSR double-blind, placebo-controlled trial. N Engl J Med 326: 1043-1049. [Crossref]

37. van Rossum MA, Fiselier TJ, Franssen MJ, Zwinderman AH, ten Cate R, et al. (1998) Sulfasalazine in the treatment of juvenile chronic arthritis: a randomized, double-blind, placebo-controlled, multicenter study. Arthritis \& Rheum 41: 808-16. [Crossref]

38. Hochberg M, Silman A, Smolen J, Weinblatt M, Weisman M (2010) Rheumatology Mosby Elsevier. Philadelphia.

39. Ravelli A, Magni-Manzoni S, Pistorio A, Besana C, Foti T, et al. (2005) Preliminary diagnostic guidelines for macrophage activation syndrome complicating systemic juvenile idiopathic arthritis. J Paediatr 146: 598-604. [Crossref]

40. Wallace C, Levinson J (1991) Juvenile rheumatoid arthritis: outcome and treatment for the 1990s. Rheum Dis Clin North Am 17: 891-905. [Crossref]

41. Minden K, Niewerth M, Listing J, Biederm ann T, Bollow M, et al. (2002) Long-term outcome in patients with juvenile idiopathic arthritis. Arthritis Rheum 46: 2392-2401. [Crossref]

Copyright: (C2017 Tekelı AA. This is an open-access article distributed under the terms of the Creative Commons Attribution License, which permits unrestricted use, distribution, and reproduction in any medium, provided the original author and source are credited. 